\title{
UNIDADES FOTOTÉRMICAS E TEMPERATURA-BASE INFERIOR DE FRUTOS DE MANGUEIRA ALFA, NA BAIXADA CUIABANA ${ }^{1}$
}

\author{
MARCELO PAES DE BARROS ${ }^{2}$, VITOR BACARIN ZANETTI ${ }^{3}$, \\ CAMILA ISABEL DE MENEZES FRAGA ${ }^{4}$, PAULO CELSO DO COUTO NINCE ${ }^{5}$, \\ JOSÉ HOLANDA CAMPELO JÚNIOR ${ }^{6}$, FRANCISCO DE ALMEIDA LOBO
}

RESUMO - O objetivo deste trabalho foi determinar a temperatura-base inferior $(\mathrm{Tb})$ a partir da unidade fototérmica (UF) para o cultivo experimental de manga Alfa sob condições de cerrado. Foram utilizados dados diários da temperatura do ar disponibilizados pela Estação Agrometeorológica Padre Ricardo Remetter, localizada em Santo Antônio do Leverger-MT $\left(15,8^{\circ} \mathrm{S}\right.$ e $\left.56,1^{\circ} \mathrm{W}, 140 \mathrm{~m}\right)$, e avaliação sensorial do estádio de maturação dos frutos de manga. A Tb foi determinada pelo método da menor variabilidade das unidades fototérmicas (UF) acumuladas do período da floração à colheita dos frutos, variabilidade avaliada pelo coeficiente de variação (cv) simulado para diferentes valores da Tb. De julho a novembro de 2007, em três plantas de um pomar demonstrativo irrigado, foram identificados 82 frutos para o acompanhamento do crescimento e maturação. Entre esses frutos, em meados de dezembro de 2007, foi possível identificar 13 frutos que atingiram a maturidade fisiológica, após um período médio de observação de 112 dias. Para exigência fototérmica de $1.878 .166,1 \mathrm{UF}$, encontrou-se $\mathrm{Tb}$ de $10^{\circ} \mathrm{C}$, valor consistente com os apresentados na literatura para a cultivar de manga, o que comprova a eficiência do método que combina a ação da temperatura e do fotoperíodo sobre a maturação dos frutos e que confere um caráter mais racional que o método tradicional da soma térmica.

Termos para indexação: Maturação, manga, cerrado, graus-dia.

\section{PHOTOTHERMAL UNITS AND LOWER BASE TEMPERATURE FOR ALFA MANGO FRUIT IN THE BAIXADA CUIABANA}

\begin{abstract}
The aim of this study was to determine the lower base temperature ( $\mathrm{Tb}$ ) for Alfa mango fruit, using the photothermal units (PTU) method in the cerrado conditions. The meteorological data for the study were acquired from "Padre Ricardo Remetter" meteorological station located at Santo Antonio do Leverger, MT, Brazil $\left(15,8^{\circ} \mathrm{S}\right.$ and $\left.56,1^{\circ} \mathrm{W}, 140 \mathrm{~m}\right)$, and the fruit data was acquired by sensorial evaluation of mango fruit maturation state. The Tb was determined by less variability of the coefficient of variation (cv) method applied to the PTU sum, from flowering to harvest, simulated for different values of $\mathrm{Tb}$. In the period from July to November of 2007, in tree plants from an irrigated experimental field, 82 fruits were marked to observe the growth and the maturation. From the marked fruits, 13 fruits were identified at the harvest point, in December of 2007, after an average observation period of 112 days. For a photothermal requirement of $1,878,166.13 \mathrm{PTU}$ it was observed a Tb for the fruits of $10^{\circ} \mathrm{C}$, this value is consistent with those reported in the literature, which proves the efficiency of the method that combines the action of the temperature and photoperiod on fruit maturity and providing a more rational method than the traditional heat units method. Index terms: Maturation, mango fruit, cerrado, degree-days.
\end{abstract}

\footnotetext{
${ }^{1}$ (Trabalho 105-09). Recebido em: 24-04-2009. Aceito para pubilcação em: 20-01-2010.

${ }^{2}$ Doutorando em Física Ambiental, Programa de Pós-Graduação em Física Ambiental, Universidade Federal de Mato Grosso, CuiabáMT,mpb9@terra.com.br

${ }^{3}$ Mestre em Física Ambiental, PPFA, UFMT, Cuiabá-MT, vitorz@gmail.com

${ }^{4}$ Mestre em Física Ambiental, PPFA, UFMT, Cuiabá-MT, camilafraga@gmail.com

${ }^{5}$ Mestre em Física Ambiental, PPFA, UFMT, Cuiabá-MT, pnince@ig.com.br

${ }^{6}$ Professor Titular, Depto. de Solos e Engenharia Rural, FAMEV/UFMT, Cuiabá-MT, campelo@cpd.ufmt.br

${ }^{7}$ Professor Adjunto, Depto. de Solos e Engenharia Rural, FAMEV/UFMT, Cuiabá-MT, f_a_lobo@cpd.ufmt.br
} 


\section{INTRODUÇÃO}

Os aspectos de crescimento e desenvolvimento da mangueira são direta e indiretamente influenciados por fatores ambientais. Segundo Cunha et al. (1994), dependendo da região produtora, a duração do período de crescimento da manga até a sua maturação, do florescimento à colheita, varia de 100 a 150 dias. As diferenças entre os ciclos de maturação das frutas na planta podem ter como causa as oscilações na temperatura do ar em cada região (WANG; ENGEL, 1998; STRECK, 2002).

Em função do tempo para a manga ser consumida ou industrializada, define-se um grau de maturidade ideal para a colheita. Frutos completamente maduros são colhidos para utilização imediata, enquanto para transporte ou armazenagem por períodos longos, frutos são colhidos no estádio maturo, a fim de chegarem ao mercado varejista em bom estado de conservação e maturação (MEDINA, 1996).

Para determinar a maturidade do fruto da mangueira, existem alguns parâmetros indicados na literatura, tais como: índices com bases em aspectos externos, em aspectos físicos e na composição química durante a época da colheita, com resultados que variam conforme a cultivar estudada (LUCENA, 2006). Apesar de subjetivos, métodos de coloração externa do fruto apresentam maiores possibilidades de uso. Morais et al. (2002) encontraram alta correlação entre o método de avaliação da cor da casca através de escala subjetiva com outros parâmetros de maturação do fruto de manga.

Uma forma para se estimar o tempo necessário para o desenvolvimento do fruto após a floração, são as unidades térmicas (graus-dia). No Brasil, diversos são os estudos que procuram determinar o ponto de colheita de frutos baseando-se na soma das unidades térmicas (MORAIS et al., 2002; LUCENA, 2006; VOLPE et al., 2002; ROBERTO et al., 2004; SILVA, 1996).

A estimativa do tempo de maturação de um fruto através da soma das unidades térmicas depende do conhecimento da temperatura-base inferior $(\mathrm{Tb})$ da cultivar, temperatura abaixo da qual seu crescimento é desprezível ou nulo, e da necessidade de que exista relação linear entre o desenvolvimento e a temperatura média do ar (ARNOLD, 1959; BRUNINI et al., 1976; CAMARGO et al., 1987; SOUZA et al., 2009).

No entanto, em algumas situações, a relação linear de dependência é baixa pelo fato de não se considerar a ação do fotoperíodo, como fator não apenas de modificação dos efeitos da temperatura, mas também indutor do desenvolvimento reprodu- tivo (VILLA NOVA et al., 2007). Robertson (1968) desenvolveu um modelo de taxa de desenvolvimento relacionado à temperatura e fotoperíodo, usando para tanto uma função quadrática para explicar os efeitos não lineares dessas variáveis sobre a taxa de desenvolvimento de trigo. O efeito do fotoperíodo foi avaliado com sucesso por Villa Nova et al. (1983), que assumiram, além da temperatura média do ar, o efeito do fotoperíodo como fator decisivo para o desenvolvimento, propondo um método alternativo baseado numa variável denominada Unidade Fototérmica (UF) para a modelagem do crescimento estacional da cana-de-açúcar sobre a produção de massa seca.

No Estado de Mato Grosso, apesar de apresentar condições satisfatórias para o cultivo, a exploração da manga ocorre apenas em regime extrativista, não tendo uma participação considerável no mercado nacional do fruto (VALENTE et al., 1996). As cultivares mais plantadas no território nacional são a 'Tommy Atkins' e a 'Keitt' (LUCENA, 2006). $\mathrm{Na}$ Baixada Cuiabana, apesar do predomínio da variedade 'Bourbon', outras cultivares estão em testes, dentre as quais a 'Alfa' e a 'Roxa', híbridas lançadas em 1998 pela Embrapa Cerrados, que foram as mais apreciadas (Valente et al., 2004).

Este trabalho tem por objetivo determinar a exigência térmica da manga 'Alfa' em unidades fototérmicas e a temperatura-base inferior da cultivar na Baixada Cuiabana.

\section{MATERIAL E MÉTODOS}

O experimento foi realizado em mangueiras de um campo demonstrativo existente na Fazenda Experimental da UFMT, no município de Santo Antônio de Leverger-MT, próximo das coordenadas de $15^{\circ} 47^{\prime}$ sul, e $56^{\circ} 04^{\prime}$ oeste, e altitude de $140 \mathrm{~m}$, distante $33 \mathrm{~km}$ de Cuiabá-MT, na microrregião da Baixada Cuiabana.

Conforme a classificação de Köeppen, a área de estudos está sob domínio climático Aw, com estação chuvosa de outubro a março e período seco de abril a setembro. A vegetação predominante é o cerrado, e a região apresenta solos pouco desenvolvidos, em consequência da exposição recente, com ocorrência de solos litólicos distróficos, às vezes álicos, raros eutróficos, e solos concrecionários (Plintossolos) distróficos, às vezes epicascalhentos.

As amostragens foram efetuadas em uma área de cerca de um hectare de um campo demonstrativo onde existem mangueiras das cultivares 'Alfa', 'Roxa' e 'Maling', transplantadas para o lugar em 
2003, dispostas no espaçamento de 6 × 8 m.

No presente estudo, foram selecionadas três plantas irrigadas de mangueira variedade 'Alfa'. A irrigação foi realizada diariamente, durante uma hora, por microaspersão, com um microaspersor por planta, e uma dose de rega de aproximadamente 30 L.planta $^{-1}$.

Durante o pleno florescimento das mangueiras, a partir de julho de 2007, tiveram início a instalação e a condução do estudo. A cada semana, foram etiquetados ao acaso tantos frutos novos quantos foram encontrados, até o número máximo de 10 frutos novos (de comprimento e diâmetro iguais ou inferiores a $15 \mathrm{~mm}$ ), distribuídos entre as três plantas. Acompanhados e medidos quinzenalmente, as avaliações se estenderam até o amadurecimento dos últimos frutos, em janeiro de 2008. Do total de 82 frutos registrados ao longo do período, apenas 13 destes vingaram e foram colhidos maduros.

Assim, apenas para acompanhar os estádios de maturação dos frutos etiquetados, foi estabelecida uma escala própria para as avaliações ( 0 - o fruto está verde crescendo; 1 - o fruto parou de crescer; 2- o fruto cede ao tato; 3 - o fruto começou a mudar de cor; 4- o fruto desapareceu; 5- o fruto mudou completamente de cor; 6- o fruto apresenta bicadas de pássaros; 7- o fruto apresenta pintas pretas; 8- o fruto está enrugado; 9- o fruto caiu de maduro) e, por meio de observações sensoriais da forma, coloração, aspecto geral e da firmeza dos frutos, os exemplares maduros foram colhidos.

Os dados diários da temperatura do ar foram obtidos por meio do registro das observações meteorológicas de rotina efetuadas na Estação Agrometeorológica Padre Ricardo Remetter, que integra a rede do $9^{\circ}$ DISME/INMET, instalada a aproximadamente $1 \mathrm{~km}$ do local do experimento.

Para a determinação da $\mathrm{Tb}$, foram escolhidos, a priori, valores inteiros de temperaturas entre $1{ }^{\circ} \mathrm{C}$ e $17^{\circ} \mathrm{C}$, que foram utilizados para o cálculo das UFs referentes ao período de maturação de cada fruto colhido. Considerando que não tenha ocorrido déficit hídrico para as plantas em questão, as UFs foram determinadas pela aplicação da equação (1) (VILLA NOVA et al., 1999):

$$
\mathrm{UF}=\frac{\left(\frac{\mathrm{n}}{2} \mathrm{GD}\right)^{\frac{\mathrm{Nf}}{\mathrm{Ni}_{\mathrm{i}}+1}}}{\frac{\mathrm{N}_{\mathrm{f}}}{\mathrm{N}_{\mathrm{i}}}+1}
$$

Em que:

UF $=>$ Número de unidades fototérmicas acumuladas no período; $\mathrm{n}=>$ Número de dias no período;

$\mathrm{N}_{\mathrm{f}}=>$ Duração astronômica do dia (horas e décimos de horas) no final do período de crescimento, e

$\mathrm{N}_{\mathrm{i}}=>$ Duração astronômica do dia (horas e décimos) no início do período de crescimento.

Sendo as duas últimas variáveis determinadas pelas relações:

$$
\begin{aligned}
& \mathrm{N}_{\mathrm{f}} \text { ou } \mathrm{N}_{i}=0,133 \cdot \operatorname{arcos}(-\operatorname{tg} \delta \cdot \operatorname{tg} \varphi) \\
& \delta=23,45 \cdot \operatorname{sen}\left[\left(\frac{360}{365}(\mathrm{Dj}-80)\right)\right]
\end{aligned}
$$

Nas equações (2) e (3), $\delta$ corresponde ao valor da declinação solar para aquela latitude, $\varphi$ a latitude do local e Dj o dia Juliano, contagem de dias corridos a partir do início do ano correspondente ao início e fim do período de crescimento e maturação do fruto.

O cálculo de graus-dia, desde a floração até a colheita, seguiu as equações propostas por Ometto (1981):

$$
\begin{aligned}
& T_{M}<T_{b}: \quad G D=0 \\
& T_{m}>T_{b}: G D=\left(T_{m}-T_{b}\right)+\left(\frac{T_{M}-T_{m}}{2}\right) \\
& T_{m}<T_{b}: G D=\frac{\left(T_{M}-T_{b}\right)^{2}}{2\left(T_{M}-T_{m}\right)} \\
& T_{m}>T_{b} e T_{B}<T_{M}: \\
& G D=\frac{2\left(T_{M}-T_{m}\right)\left(T_{m}-T_{b}\right)+\left(T_{M}-T_{m}\right)^{2}-\left(T_{M}-T_{B}\right)^{2}}{2\left(T_{M}-T_{m}\right)} \\
& T_{m}<T_{b} e T_{B}<T_{M}: \\
& \quad G D=\frac{1}{2}\left[\frac{\left(T_{m}-T_{b}\right)^{2}-\left(T_{M}-T_{B}\right)^{2}}{\left(T_{M}-T_{m}\right)}\right]
\end{aligned}
$$

em que:

$G D=>$ Graus-dia;

$\mathrm{T}_{\mathrm{M}}=>$ Temperatura máxima diária $\left({ }^{\circ} \mathrm{C}\right)$;

$\mathrm{T}_{\mathrm{m}}^{\mathrm{M}} \Rightarrow>$ Temperatura mínima diária $\left({ }^{\circ} \mathrm{C}\right)$;

$\mathrm{T}_{\mathrm{b}} \Rightarrow>$ Temperatura-base inferior $\left({ }^{\circ} \mathrm{C}\right)$, e

$\mathrm{T}_{\mathrm{B}}=>$ Temperatura-base superior $\left({ }^{\circ} \mathrm{C}\right)$.

Posteriormente, para cada $\mathrm{Tb}$ simulada, foi determinado o coeficiente de variação $(\mathrm{cv})$, resultado da razão entre o desvio-padrão da UF e o valor médio desta variável para os frutos colhidos, sendo a $\mathrm{Tb}$ da cultivar aquela que correspondeu ao menor valor do cv.

$\mathrm{O}$ cv é uma forma de expressar a variabili- 
dade dos dados, minimizando a influência da ordem de grandeza da variável, de forma que quanto menor o valor do cv mais homogêneo é o conjunto.

\section{RESULTADOS E DISCUSSÃO}

Para os valores obtidos relativos à maturação da manga alfa irrigada, os dados astronômicos de fotoperíodos inicial e final $\left(\mathrm{N}_{\mathrm{i}} \mathrm{e} \mathrm{N}_{\mathrm{f}}\right)$, temperatura do ar (T) e duração do período em dias (N), para cada amostra, foram ajustados desconsiderando amostras repetidas, conforme relatado na Tabela 1.

Para a determinação da temperatura-base foi considerada a faixa de temperaturas de 1 a 17 ${ }^{\circ} \mathrm{C}$. Com o ajuste dos valores observados para os coeficientes de variação das UFs, cujo valor de $\mathrm{Tb}$ é aquele que corresponde ao menor cv (Figura 1), verificou-se que a $\mathrm{Tb}$ para o ciclo de manga 'Alfa' na Baixada Cuiabana foi de $10{ }^{\circ} \mathrm{C}$ e que, para tal temperatura-base, a cultivar apresentou a exigência de 1.878.166,1 UF, do início da frutificação até a colheita.

$\mathrm{O}$ valor encontrado para a $\mathrm{Tb}$ verificada para o ciclo de manga 'Alfa', conforme o método das unidades fototérmicas, é coerente com a faixa citada por Cooper \& Tainton (1968), de 10 a $15{ }^{\circ} \mathrm{C}$ como referência para plantas tropicais, e idêntico ao valor encontrado por Jacques (1994), de $10{ }^{\circ} \mathrm{C}$ para as mesmas espécies.

Lucena (2006), em trabalho realizado com mangas da variedade 'Tommy Atkins' cultivadas no vale do São Francisco, em Petrolina-PE, sugere o valor de $10{ }^{\circ} \mathrm{C}$ como a temperatura-base da mangueira para a região. Outros trabalhos realizados com frutos de manga sugerem o valor $10{ }^{\circ} \mathrm{C}$ como a temperatura-base da mangueira (CHAUDHRI, 1976 ; SILVA, 1996).

$\mathrm{O}$ valor médio das unidades térmicas acumuladas para o ciclo da manga 'Alfa' na Baixada Cuiabana foi de $2116,5{ }^{\circ} \mathrm{C}$. Souza et al. (2007), estudando a curva do crescimento de frutos de mangueira em uma área de produção comercial, em Janaúba-MG, considerando uma temperatura-base inferior de $10^{\circ} \mathrm{C}$, encontraram valores de somas térmicas de $1.270,2^{\circ} \mathrm{C}, 1.962,7^{\circ} \mathrm{C}$ e $1.627,5^{\circ} \mathrm{C}$ para as cultivares 'Haden', 'Palmer' e 'Tommy Atkins', respectivamente. Mosqueda-Vasquez e Ireta-Ojeda (1993), em trabalho sobre a maturidade do fruto da mangueira 'Manila', cultivada no México, verificaram a necessidade de $2.292,7^{\circ} \mathrm{C}$ para a maturidade do fruto ser atingida com a $\mathrm{Tb}$ de $0,33{ }^{\circ} \mathrm{C}$.

$\mathrm{O}$ teste de aderência de Kolmogorov-Smirnov revelou a distribuição normal, em nível de significância de 0,20 , como adequada ao estudo para representar o período em dias de maturação da manga, estimando os parâmetros (média e desvio-padrão) desta distribuição. O período de maturação médio da manga variedade 'Alfa' obtido neste trabalho foi de 112 dias para atingir o ponto de colheita. O intervalo de confiança da média para um nível de significância de 0,05 ficou entre 102 e 121 dias, que é superior aos 96 dias após a floração plena observados por Morais et al. (2002), no polo Mossoró-Açu, no Rio Grande do Norte, estudando a variedade 'Tommy Atkins'.

Os valores encontrados neste trabalho são superiores também aos de Lucena (2006), que observou que as mangas da variedade 'Tommy Atkins' cultivadas no vale do São Francisco, em Petrolina-PE, que atingiram a maturidade fisiológica aos 98 dias após a antese (DAA). Para a mesma região, estudando a variedade 'Haden', Castro Neto e Reinhardt (2003) constataram que, 75 dias após a floração, ocorreu o máximo acúmulo de massa seca e que este período pode ser considerado como necessário para que o fruto da manga atinja a maturação. 
TABELA 1- Início da observação da cultivar de manga Alfa na Baixa Cuiabana até o amadurecimento do fruto, período em dias $(\mathrm{N})$, temperatura do ar mínima $\left(\mathrm{T}_{\min }\right)$ e máxima $\left(\mathrm{T}_{\text {máx }}\right)$, fotoperíodo inicial (Ni) e final (Nf), graus-dia (GD) e unidades fototérmicas (UF) para uma temperaturabase inferior de $10{ }^{\circ} \mathrm{C}$.

\begin{tabular}{|c|c|c|c|c|c|c|c|c|c|}
\hline \multirow{2}{*}{ Fruto } & \multicolumn{2}{|c|}{ Marcação } & \multirow{2}{*}{$\mathrm{N}$} & \multicolumn{2}{|c|}{$\begin{array}{c}\text { Temperatura } \\
\text { do ar }\left({ }^{\circ} \mathrm{C}\right)\end{array}$} & \multirow{2}{*}{$\mathrm{Ni}(\mathrm{h})$} & \multirow{2}{*}{$\mathrm{Nf}(\mathrm{h})$} & \multirow{2}{*}{$\begin{array}{l}\text { GD } \\
\left({ }^{\circ} \mathrm{C}\right)\end{array}$} & \multirow{2}{*}{ UF } \\
\hline & Inicial & Maduro & & $\mathrm{T}_{\text {min }}$ & $\mathrm{T}_{\max }$ & & & & \\
\hline 1 & 02-08-07 & 26-11-07 & 117 & 24,4 & 33,3 & 11,3 & 12,8 & 2203,1 & 2700245,6 \\
\hline 2 & $20-08-07$ & $16-12-07$ & 119 & 25,2 & 33,2 & 11,5 & 12,9 & 2280,6 & 2643407,4 \\
\hline 3 & $27-08-07$ & $05-01-08$ & 132 & 25,4 & 33,0 & 11,6 & 12,8 & 2531,8 & 3047497,9 \\
\hline 4 & 03-09-07 & 05-01-08 & 125 & 25,7 & 33,2 & 11,7 & 12,8 & 2429,9 & 2636518,5 \\
\hline 5 & 03-09-07 & $12-01-08$ & 132 & 25,7 & 33,1 & 11,7 & 12,8 & 2556,1 & 2868636,1 \\
\hline 6 & 17-09-07 & $22-12-08$ & 95 & 25,7 & 32,4 & 11,9 & 12,9 & 1826,3 & 1321348,7 \\
\hline 7 & $17-09-07$ & $19-01-08$ & 122 & 25,5 & 32,2 & 11,9 & 12,8 & 2313,4 & 2018331,0 \\
\hline 8 & $17-09-07$ & $26-01-08$ & 129 & 25,5 & 32,7 & 11,9 & 12,7 & 2428,8 & 2158867,7 \\
\hline 9 & 08-10-07 & 05-01-08 & 87 & 25,4 & 32,1 & 12,3 & 12,8 & 1627,7 & 873412,0 \\
\hline 10 & $08-10-07$ & $12-01-08$ & 97 & 25,3 & 31,9 & 12,3 & 12,8 & 1801,6 & 1054681,3 \\
\hline 11 & 08-10-07 & 19-01-08 & 104 & 25,3 & 31,8 & 12,3 & 12,8 & 1929,0 & 1181936,9 \\
\hline 12 & $15-10-07$ & 19-01-08 & 94 & 25,2 & 31,7 & 12,4 & 12,8 & 1735,5 & 907795,5 \\
\hline 13 & $15-10-07$ & $26-01-08$ & 101 & 25,2 & 31,5 & 12,4 & 12,7 & 1851,0 & 1003481,2 \\
\hline
\end{tabular}

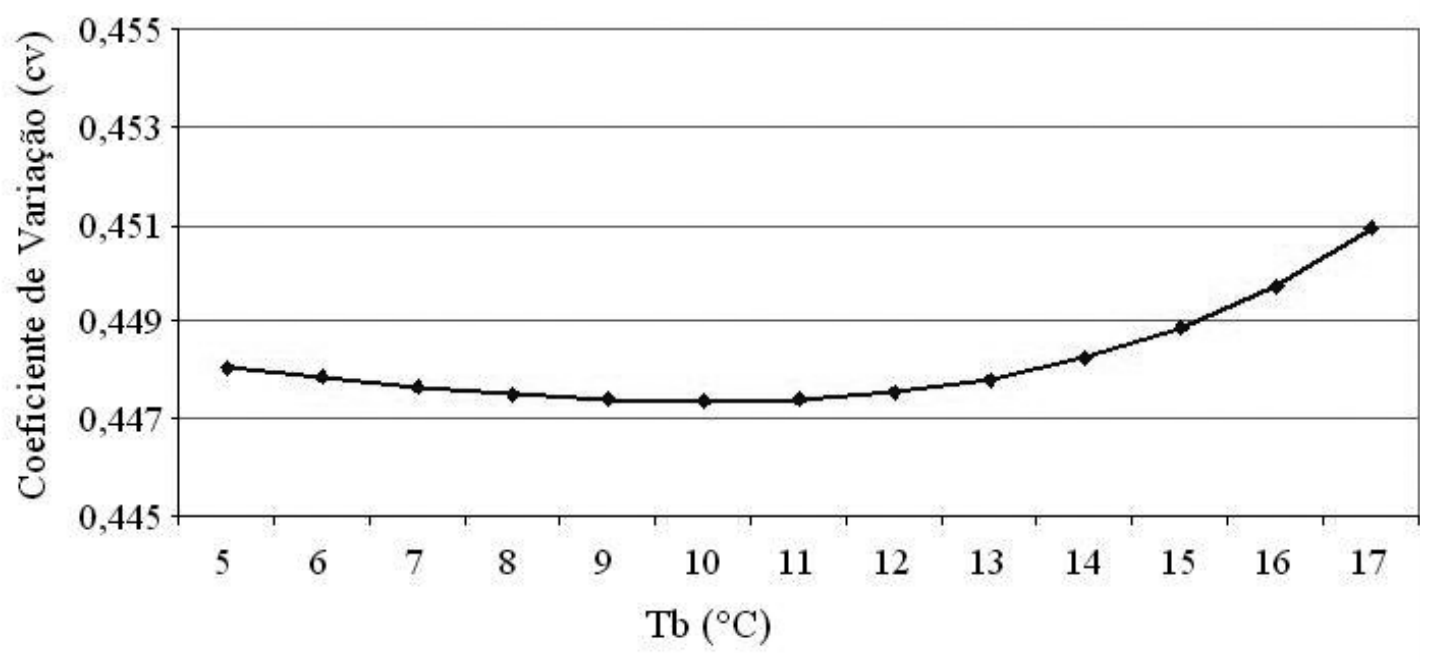

$\rightarrow$ Coeficientes de Variação das Unidades Fototérmicas (UF)

FIGURA 1 - Coeficiente de variação (cv) das Unidades Fototérmicas pela Temperatura-Base Inferior (Tb) em ${ }^{\circ} \mathrm{C}$ para a cultivar de manga 'Alfa' na Baixada Cuiabana, no período de junho de 2007 a janeiro de 2008. 


\section{CONCLUSÕES}

1-A temperatura-base inferior para o período que se estende do início da frutificação até a maturação dos frutos da mangueira 'Alfa' é de $10^{\circ} \mathrm{C}$.

2-Nas condições de cerrado da Baixada Cuiabana, em média, os frutos atingem o ponto de colheita aos 112 dias após a frutificação.

3-A soma térmica, expressa em graus-dia, como média do ciclo analisado, é de $2116,5^{\circ} \mathrm{C}$.

4-A exigência fototérmica média, do início da frutificação até a colheita, é de 1.878.166,1 UF.

5. A unidade fototérmica permite estimar com boa aproximação o valor da temperatura-base inferior, sendo uma variável climática que combina a ação da temperatura e do fotoperíodo sobre a maturação dos frutos e que confere ao método um caráter mais racional que o método tradicional da soma térmica.

\section{AGRADECIMENTOS}

À equipe da Estação Agrometeorológica Padre Ricardo Remetter, pela colaboração com os dados micrometeorológicos.

\section{REFERÊNCIAS}

ARNOLD, C. Y. The determination and significance of the base temperature in a linear heat unit system. Proceedings America Society for Horticulture Science, Virginia, v.74, p.430-445, 1959.

BRUNINI, O.; LISBÃO, R. S.; BERNARDI, J. B.; FORNASIER, J. B.; PEDRO JÚNIOR, M. J. Temperatura-base para alface cultivar "White Boston", em um sistema de unidades térmicas. Bragantia, Campinas, v.35, n.19, p. 213-219, 1976.

CAMARGO, M. B. P.; BRUNINI, O.; MIRANDA, M. A. C. Temperatura-base para cálculo dos grausdia para cultivares de soja em São Paulo. Pesquisa Agropecuária Brasileira, Brasília, v.22, n.2, p.115$121,1987$.

CASTRO NETO, M. T.; REINHARDT, D. H. Relações entre parâmetros de crescimento do fruto de manga cv. Haden. Revista Brasileira de Fruticultura, Jaboticabal, v.25, n.1, p.35-37, 2003.
CHAUDHRI, S. A. Mango. In: GARDNER, R. S.; CHAUDHRI, S. A. The propagation of tropical fruits trees. England: CAB International, 1976. p.403-474.

COOPER, J. P.; TAINTON, N. M. Light and temperature requirements for the growth of tropical and temperate grasses. Review article. Herbage Abstracts, Farnham Royal, v.38, p.167-176, 1968.

CUNHA, G. A. P.; SAMPAIO, J. M. M.; NASCIMENTO, A. S.; SANTOS FILHO, H. P.; FONSECA, N. A cultura da manga. Brasília: Embrapa-SPI, 1994. 54p.

JACQUES, A. V. A. Caracteres morfofisiológicos e suas implicações com o manejo. In: CARVALHO, M. M.; ALVIM, M. J.; XAVIER, D.F. (Ed.). Capimelefante: produção e utilização. Coronel Pacheco: EMBRAPA\CNPGL, 1994. p.31-48.

LUCENA, E. M. P. Desenvolvimento e maturidade fisiológica de manga 'Tommy Atkins' no Vale do São Francisco. 2006. 152 f. Tese (Doutorado em Agronomia) - Universidade Federal do Ceará, Fortaleza, 2006.

MEDINA, V. M. Fisiologia e Pós-Colheita da Manga. In: JOSÉ, A. R. S.; SOUZA I. V. B.; MARTINS FILHO, J.; MORAIS, O. M. (Org.). Manga: tecnologia de produção e mercado. Vitória da Conquista: Universidade do Sudoeste da Bahia, 1996. p. 202-222.

MORAIS, P. L. D.; FILGUEIRAS, H. A. C.; PINHO, L. N.; ALVES, R. E. Ponto de colheita ideal de manga 'Tommy Atkins' para o mercado europeu. Revista Brasileira de Fruticultura, Jaboticabal, v.24, n.3, p.671-675, 2002.

MOSQUEDA-VÁZQUEZ, R.; IRETA-OJEDA, A. Degree-days and base temperatures required for inflorescence and fruit development in mango 'Manila'. Acta Horticulturae, Leuven, n.341, p. 232-237, 1993.

OMETTO, J. C. Bioclimatologia vegetal. São Paulo: Ceres, 1981. 435p. 
ROBERTO, S. R.; SATO, A. J.; BRENNER, E. A.; SANTOS, C. E.; GENTA, W. Fenologia e soma térmica (graus-dia) para a videira 'Isabel' (Vitis labrusca) cultivada no noroeste do Paraná. Semina: Ciências Agrárias, Londrina, v.25, n.4, p. 273-280, 2004.

ROBERTSON, G. W. A biometeorological time scale for a cereal crop involving day and night temperatures and photoperiod. International Journal Biometeorology, Lisse, v.12, p.191-223, 1968.

SILVA, J. S. O. Produção de manga: manual. Viçosa: CPT, 1996. 34p.

SOUZA, A. P.; SILVA, A. C.; LEONEL, S.; ESCOBEDO, J. F. Temperaturas basais e soma térmica para a figueira podada em diferentes épocas. Revista Brasileira de Fruticultura, Jaboticabal, v.31, n.2, p.314-322, 2009.

SOUZA, F. V. Curva de crescimento e exportação de nutrientes e sódio por frutos de mangueira Palmer, Haden e Tommy Atkins. 2007. 54 f. Dissertação (Mestrado em Agronomia) - Universidade Estadual Paulista, Jaboticabal, 2007.

STRECK, N. A. A generalized nonlinear air temperature response function for node appearence rate in muskmelon (Cucumis melo L.). Revista Brasileira de Agrometeorologia, Santa Maria, v.10, n.1, p.105$111,2002$.

VALENTE, J. P.; DALDEGAN, L. H. C.; SANTOS, V. J.; AMARAL, E. T. Indução de floração em mangueira cv. Borbon na baixada cuiabana com nitrato de potássio $\left(\mathrm{KNO}_{3}\right)$. In: CONGRESSO BRASILEIRO DE FRUTICULTURA, 16., 1996, Curitiba. Fruticultura Sem Fronteiras... Londrina: IAPAR, 1996. v.1, p.302-302.
VALENTE, J. P.; QUINTINO, A. C.; SILVA, E. L. Fenologia de espécies frutíferas nas condições da Baixada Cuiabana. In: ENCONTRO DE INICIAÇÃO CIENTÍFICA, 12., 2004, Cuiabá. Palestras... Cuiabá: EdUFMT, 2004. v.1, p.187-187.

VILLA NOVA, N.A.; BARIONI, L.G.; PEDREIRA, C.G.S.; PEREIRA, A.R. Modelo para a previsão da produtividade do capim-elefante em função de temperatura do ar, fotoperíodo e frequência de desfolha.

Revista Brasileira de Agrometeorologia, Santa Maria, v.7, n.1, p.75-79, 1999.

VILLANOVA, N. A.; CARRETEIRO, M. V.; SCARDUA, R. Um modelo de avaliação do crescimento de cana-de-açúcar (Saccharum spp.) em termos da ação combinada do fotoperíodo e da temperatura média do ar. In: CONGRESSO BRASILEIRO DE AGROMETEOROLOGIA, 1983, Campinas. Anais... Campinas: Sociedade Brasileira de Agrometeorologia, 1983. p.31-48.

VILLA NOVA, N.A.; TONATO, F.; PEDREIRA, C. G. S.; MEDEIROS, H. R. Método alternativo para cálculo da temperatura-base de gramíneas forrageiras. Ciência Rural, Santa Maria, v.37, n.2, p.545-549, 2007.

VOLPE, C. A.; SCHÖFFEL, E. R.; BARBOSA, J. C. Influência da Soma Térmica e da Chuva durante o desenvolvimento de laranjas 'Valência' e 'Natal' na relação entre Sólidos Solúveis e Acidez e no Índice Tecnológico do Suco. Revista Brasileira de Fruticultura, Jaboticabal, v.24, p.436-441, 2002.

WANG, E.; ENGEL, T. Simulation of phenological development of wheat crops. Agricultural Systems, London, v.58, p.1-24, 1998. 